\title{
Tilted- and Axial-Beam Formation by a Single-Arm Rectangular Spiral Antenna With Compact Dielectric Substrate and Conducting Plane
}

\author{
Hisamatsu Nakano, Fellow, IEEE, Jun Eto, Yosuke Okabe, and Junji Yamauchi, Member, IEEE
}

\begin{abstract}
A single-arm rectangular spiral antenna is analyzed using the finite-difference time-domain method. The spiral is printed on a finite-size dielectric substrate backed by a finite-size conducting plane. Both the substrate and conducting planes are square with a side length $L$ of less than $0.6 \lambda_{0}\left(\lambda_{0}\right.$ : wavelength in free space). The radiation pattern is dependent on the outermost arm peripheral length $C$. The spiral whose peripheral length is within $2 \lambda_{g}<C<3 \lambda_{g}$ ( $\lambda_{g}$ : the guided wavelength of the current) radiates a tilted beam of circular polarization. When the peripheral length is decreased to $\lambda_{g}<C<2 \lambda_{g}$, the spiral radiates an axial beam. The axial beam has a wide half-power beam width of approximately $102^{\circ}$ (for $L \approx 0.369 \lambda_{0}$ ) with a gain of approximately $6.7 \mathrm{~dB}$. The axial beam shows a $15 \%$ frequency bandwidth for a 3-dB axial ratio criterion. Over this bandwidth, the voltage standing-wave ratio (VSWR) is less than two, as desired. The experimental results for the radiation pattern, gain, axial ratio, and VSWR are also presented.
\end{abstract}

Index Terms-Beam formation, finite-difference time-domain (FDTD) analysis, printed spiral antenna.

\section{INTRODUCTION}

$\mathbf{M}$ ANY spiral configurations have been investigated as radiation elements [1]-[11]. Among them, the single-arm spiral fed from a coaxial line [9] has the advantage that it does not need balun circuits between the spiral and the feed line. The single-arm spiral in [9] was analyzed under the condition that it is located in free space. The analysis was performed using an integral equation for the antenna current with a free-space Green function [12]. Note that the integral equation was solved by the method of moments (MoM) [13].

The spiral in [9] has a round configuration and is supported by a honeycomb material of relative permittivity $\varepsilon_{r} \approx 1$ (free space). The round spiral is backed by a conducting plane of infinite extent. In this paper, analysis is focused on a single-arm spiral antenna, which differs from the round spiral [9] in configuration and support material. The single-arm spiral to be analyzed is rectangular and is printed on a finite-size dielectric substrate of $\varepsilon_{r}>1$, backed by a finite-size conducting plane.

Integral equation techniques developed recently [14], [15] can predict the radiation characteristics of an antenna printed on a dielectric substrate backed by a conducting plane. However, these techniques cannot handle the practical case where

Manuscript received September 17, 1998, revised March 12, 2001.

The authors are with the College of Engineering, Hosei University, Koganei, 184-8584 Tokyo, Japan (e-mail: nakano@k.hosei.ac.jp).

Publisher Item Identifier S 0018-926X(02)01723-4. the dielectric substrate and conducting plane are of finite size. These techniques can only handle the antenna on an infinite-size dielectric substrate backed by an infinite-size conducting plane.

The single-arm rectangular spiral in this paper, therefore, is analyzed using a different method: the finite-difference time-domain (FDTD) method [16], [17]. First, the FDTD method is applied to a spiral antenna whose outermost arm peripheral length $C$ is within $2 \lambda_{g}<C<3 \lambda_{g}$ (where $\lambda_{g}$ is the guided wavelength of the current). The antenna characteristics, including the current distribution, voltage standing-wave ratio (VSWR), radiation pattern, gain, and axial ratio, are evaluated on the basis of the electric and magnetic fields obtained in the time domain. It is found that a tilted beam of circular polarization is obtained. Secondly, the FDTD method is applied to a spiral antenna whose outermost arm peripheral length $C$ is within $1 \lambda_{g}<C<2 \lambda_{g}$. The spiral is constructed with a small-size dielectric substrate and conducting plane for compactness. It is revealed that the spiral radiates an axial beam of circular polarization. Since the axial beam has many applications, the frequency responses of the gain, axial ratio, and VSWR are investigated both theoretically and experimentally.

It should be emphasized that the above FDTD analyses show that the single-arm rectangular spiral antenna is a circularly polarized element. The results of these analyses are obviously different from those for an antenna with similar shape-the two-arm rectangular spiral antenna radiating a linearly polarized wave [10].

\section{CONFIGURATION}

Fig. 1 shows the configuration of a single-arm rectangular spiral antenna. The horizontal spiral arm of strip width $w$ is printed on a square dielectric substrate and connected to a vertical arm of wire radius $\rho_{v}$. The antenna is fed by a coaxial line from point $o$.

The square dielectric substrate of permittivity $\varepsilon_{r}$ has thickness $B$ and side length $L$. The dielectric is backed by a conducting plane (ground plane), which is also square and has the same side length as the dielectric. The horizontal spiral arm is composed of multiple filaments, whose lengths are $a_{0}, a_{0}, 2 a_{0}, 2 a_{0}, 3 a_{0}, 3 a_{0}, \ldots,(M-1) a_{0},(M-1) a_{0}, M a_{0}$, and $S a_{0}$. The value $4 M a_{0}(\equiv C)$ is regarded as the outermost peripheral length of the spiral arm. 


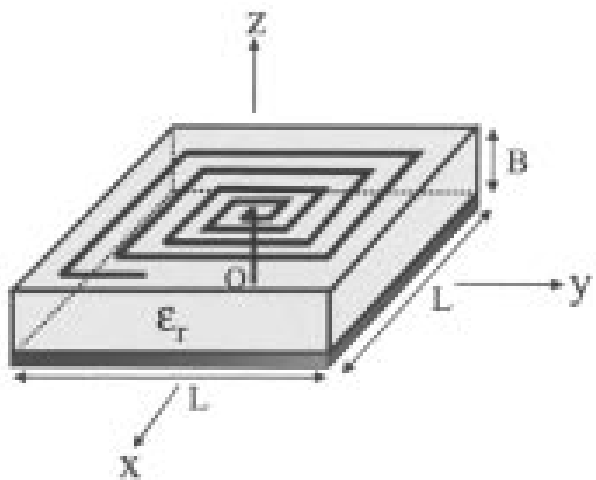

(a)

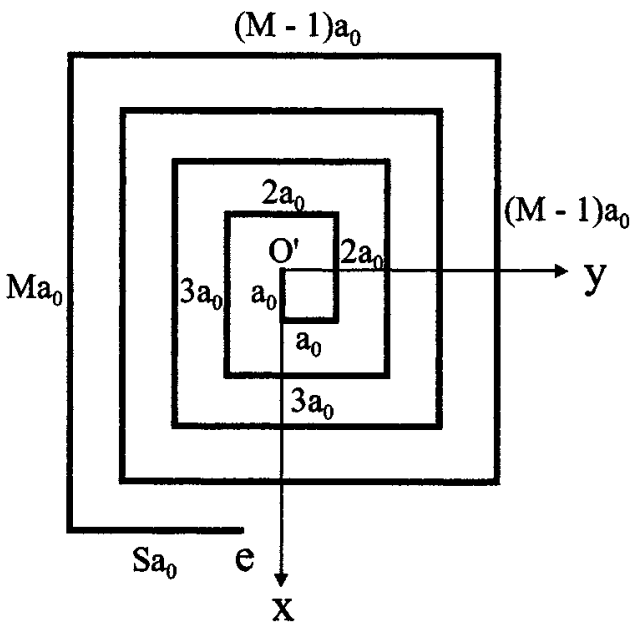

(b)

Fig. 1. Single-arm rectangular spiral antenna. (a) Perspective. (b) Arm configuration. Point $o^{\prime}$ is the starting point of a horizontal arm and located just above the origin $o$.

The following configuration parameters are fixed throughout this paper: $w=1.2 \mathrm{~mm} \approx 0.0134 \lambda_{0}, \rho_{v}=0.65 \mathrm{~mm} \approx$ $0.00726 \lambda_{0}, \varepsilon_{r}=3.7, B=12 \mathrm{~mm} \approx 0.134 \lambda_{0}$, and $a_{0}=$ $4.5 \mathrm{~mm} \approx 0.0503 \lambda_{0}$, where $\lambda_{0}(\approx 89.6 \mathrm{~mm})$ is the wavelength at a test frequency of $f_{0}=3.35 \mathrm{GHz}$.

The arm filament parameters $M$ and $S$, which determine the horizontal arm length $S_{h}$ arm from point $o^{\prime}$ to point $e$, and the side length $L$ are changed subject to the objectives of the analysis.

\section{ANALYSIS METHOD}

The FDTD method is adopted to analyze a single-arm rectangular spiral antenna. Yee's algorithm [16] is used with Liao's second-order absorbing boundary condition [18]. The cell size in the computation space is chosen to be $\Delta x=\Delta y=\Delta z \equiv$ $\Delta=1.5 \mathrm{~mm} \approx 0.0168 \lambda_{0}$, with $c \Delta t=\Delta / 2 \approx 0.00838 \lambda_{0}$, where $c$ and $\Delta t$ are the velocity of light and the time step, respectively, which satisfy Courant's stability condition [17].

The excitation is modeled by a delta-gap source as shown in Fig. 2 [19]. The $z$-component of the excitation electric field is given as $E_{z}^{n}\left(i_{f}, j_{f}, k_{f}+0.5\right)=$ $-V(n \Delta t) / \Delta z$, where $V(n \Delta t)$ is the voltage applied to the gap at time $t=n \Delta t$. A sine function modu-

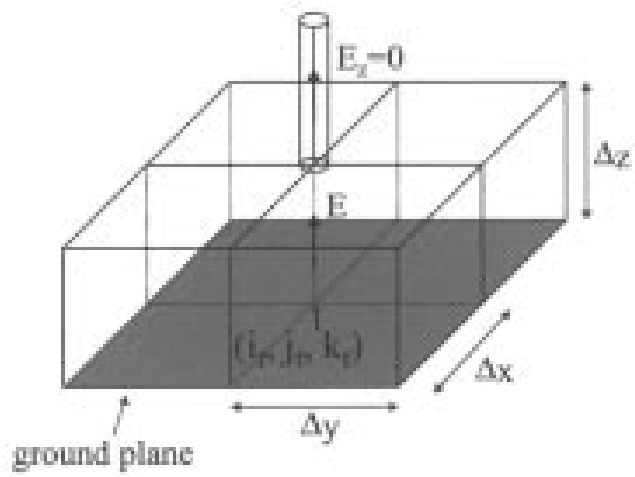

Fig. 2. Delta-gap source model for the FDTD method [19].

lated by a Gaussian function is used for this voltage: $V(n \Delta t)=\exp \left[-\{(n \Delta t-T) / 0.29 T\}^{2}\right] \sin \left(2 \pi f_{0} n \Delta t\right)$ with a constant $T=0.646 / f_{3 \mathrm{~dB}}$, where $f_{3 \mathrm{~dB}}$ is the frequency at which the power spectrum drops $3 \mathrm{~dB}$ from the maximum value.

The current along the antenna arm is obtained by integrating the magnetic field around the antenna arm (Ampere's law). The input impedance $Z_{\text {in }}=R_{\text {in }}+j X_{\text {in }}$ is given as $V(\omega) / I(\omega)$, where $V(\omega)$ and $I(\omega)$ are the input voltage and current in the frequency domain, respectively, i.e., $V(\omega)$ and $I(\omega)$ are the Fourier transforms of the time domain voltage and current $V(t)$ and $I(t)$, respectively.

The radiation field (far field) is calculated using the electric current $\mathbf{J}_{s}\left(\omega, \mathbf{R}^{\prime}\right)$ and the magnetic current $\mathbf{M}_{s}\left(\omega, \mathbf{R}^{\prime}\right)$ on a surface that encloses the antenna [19]. The $\mathbf{J}_{s}\left(\omega, \mathbf{R}^{\prime}\right)$ and $\mathbf{M}_{s}\left(\omega, \mathbf{R}^{\prime}\right)$ are, respectively, the Fourier transforms of the timedomain currents $\mathbf{J}_{s}\left(t, \mathbf{R}^{\prime}\right)$ and $\mathbf{M}_{s}\left(t, \mathbf{R}^{\prime}\right)$

$$
\begin{aligned}
\mathbf{J}_{s}\left(t, \mathbf{R}^{\prime}\right) & =\hat{n} \times \mathbf{H}\left(t, \mathbf{R}^{\prime}\right) \\
\mathbf{M}_{s}\left(t, \mathbf{R}^{\prime}\right) & =\mathbf{E}\left(t, \mathbf{R}^{\prime}\right) \times \hat{n}
\end{aligned}
$$

where $\mathbf{R}^{\prime}$ is the position vector from the coordinate origin to the point at which $\mathbf{E}\left(t, \mathbf{R}^{\prime}\right)$ and $\mathbf{H}\left(t, \mathbf{R}^{\prime}\right)$ are evaluated on the closed surface and $\hat{n}$ is the outward unit vector normal to the enclosed surface. The $\theta$ and $\phi$ components of the radiation field (in the frequency domain) at a far-field point $(R, \theta, \phi)$ are given as

$$
\begin{aligned}
& E_{\theta}(\omega)=\frac{j k_{0}}{4 \pi} \frac{e^{-j k_{0} R}}{R}\left[-Z_{0} \mathbf{N}(\omega) \bullet \hat{\theta}-\mathbf{L}(\omega) \bullet \hat{\phi}\right] \\
& E_{\phi}(\omega)=\frac{j k_{0}}{4 \pi} \frac{e^{-j k_{0} R}}{R}\left[-Z_{0} \mathbf{N}(\omega) \bullet \hat{\phi}+\mathbf{L}(\omega) \bullet \hat{\theta}\right]
\end{aligned}
$$

with

$$
\begin{aligned}
& \mathbf{N}(\omega)=\int_{\text {closed surface }} \mathbf{J}_{S}\left(\omega, \mathbf{R}^{\prime}\right) e^{j k_{0} \hat{R} \bullet \mathbf{R}^{\prime}} d S^{\prime} \\
& \mathbf{L}(\omega)=\int_{\text {closed surface }} \mathbf{M}_{S}\left(\omega, \mathbf{R}^{\prime}\right) e^{j k_{0} \hat{R} \bullet \mathbf{R}^{\prime}} d S^{\prime}
\end{aligned}
$$

where $k_{0}$ is the wave number in free space, $Z_{0}$ is the intrinsic impedance $(120 \pi \Omega)$, and $\hat{R}, \hat{\theta}$ and $\hat{\phi}$ are the unit vectors for the spherical coordinates.

After the radiation field $E_{\theta} \hat{\theta}+E_{\phi} \hat{\phi}$ is decomposed into a right-hand circularly polarized wave component $E_{R}(\hat{\theta}-j \hat{\phi})$ and a left-hand circularly polarized wave component $E_{L}(\hat{\theta}+j \hat{\phi})$, the axial ratio is calculated using $\mathrm{AR}=\left(\left|E_{R}\right|+\left|E_{L}\right|\right) /\left(\left|E_{R}\right|-\right.$ 
$\left.\left|E_{L}\right|\right)$. The $E_{\theta}, E_{\phi}, E_{R}$, and $E_{L}$ have the following relationships: $\left|E_{\theta}\right|^{2}+\left|E_{\phi}\right|^{2}=2\left\{\left|E_{R}\right|^{2}+\left|E_{L}\right|^{2}\right\}$.

The antenna in Fig. 1 is expected to radiate a right-hand circularly polarized wave due to the winding sense. The antenna gain for the right-hand circular polarization at a far-field point $(R, \theta, \phi)$ is evaluated as $G_{R}=\left|E_{R}(R, \theta, \phi)\right|^{2} R^{2} / 30 P_{\mathrm{in}}$, where $P_{\text {in }}$ is the power input to the antenna. Note that the gain $G_{R}$ decreases as the axial ratio (AR) increases in accordance with $G_{R}=G /\left[1+\{(\mathrm{AR}-1) /(\mathrm{AR}+1)\}^{2}\right]$, where $G=\left\{\left(\left|E_{\theta}\right|^{2}+\right.\right.$ $\left.\left.\left|E_{\phi}\right|^{2}\right) / 2\right\} R^{2} / 30 P_{\text {in }}$.

\section{RESULTS AND DISCUSSIONS}

The configuration in which the peripheral length $C \equiv 4 M a_{0}$ is larger than $n \lambda_{g}$ ( $n$ : integer) but smaller than $(n+1) \lambda_{g}$ is designated as the $C_{\mathbf{n},(\mathbf{n}+1)}$ configuration, where $\lambda_{g}$ is the guided wavelength of the current on the spiral. When the current along the spiral antenna travels smoothly toward the arm end "e," the currents along square loop regions on the spiral plane, each having a length of approximately $m \lambda_{g}$ ( $m$ : integer), strongly contribute to circularly polarized radiation [1]. In this section, two cases are considered: $n=2\left(C_{2,3}\right)$ and $n=1\left(C_{1,2}\right)$. Note that the $C_{2,3}$ configuration includes two square loop regions with $m=1$ and 2 . Also, note that the $C_{1,2}$ configuration includes a single square loop region with $m=1$.

\section{A. Spiral With a $C_{2,3}$ Configuration (Tilted-Beam Spiral)}

The following values are assigned to the arm filament parameters: $M a_{0}=24 \Delta(M=8)$ and $S a_{0}=2 \Delta(S \approx 0.667)$. The other configuration parameters have been defined in Section II. Note that the horizontal length measured along the spiral arm from point $o^{\prime}$ to the arm end point $e$ is given as $S_{h \text { arm }}=194 \Delta \approx 3.25 \lambda_{0}$ with a peripheral length of $C=4 M a_{0}=96 \Delta \approx 1.61 \lambda_{0} \approx 2.47 \lambda_{g, \text { rough }}$, where $\lambda_{g}$, rough is a guided wavelength roughly estimated by $\lambda_{g, \text { rough }}=\lambda_{0} /\left\{\left(\varepsilon_{r}+1\right) / 2\right\}^{1 / 2} \approx 0.652 \lambda_{0}$.

For validation, the current distribution obtained by the FDTD method is compared with that obtained by the MoM (see Fig. 3). The side length $L$ is chosen to be infinite since the MoM [14], [15] cannot handle an antenna with finite-size dielectric substrate and conducting plane. It is found that the results of both methods are in good agreement. After this validation, the side length of the dielectric substrate (= the side length of the conducting plane) is reduced to a compact size of $L=34 \Delta \approx$ $0.570 \lambda_{0}$. The results that follow (Figs. 4-8) are for this finite side length $L$.

Fig. 4 shows the current distribution. It is found that a travelling-wave current is dominant. (Note that a comparison of Figs. 3 and 4 shows a noticeable difference in the current distributions near the arm end.) The arm length from point $o^{\prime}$ to point $q_{6 \pi}$ is $166.4 \mathrm{~mm}$, yielding a phase change of $6 \pi \mathrm{rad}$. The guided wavelength (average value) is, therefore, approximated to be $\lambda_{g} \approx 0.619 \lambda_{0}$, which is close to $\lambda_{g}$, rough $\left(\lambda_{g} / \lambda_{g}\right.$, rough $\approx$ $0.95)$. It follows that the roughly estimated wavelength $\lambda_{g}$, rough gives a good estimation for the guided wavelength $\lambda_{g}$ and can be used for designing the spiral arm configuration. Thus, the peripheral length in terms of the guided wavelength is given as $C \approx 2.60 \lambda_{g}$, satisfying the condition for the $C_{2,3}$ configuration.

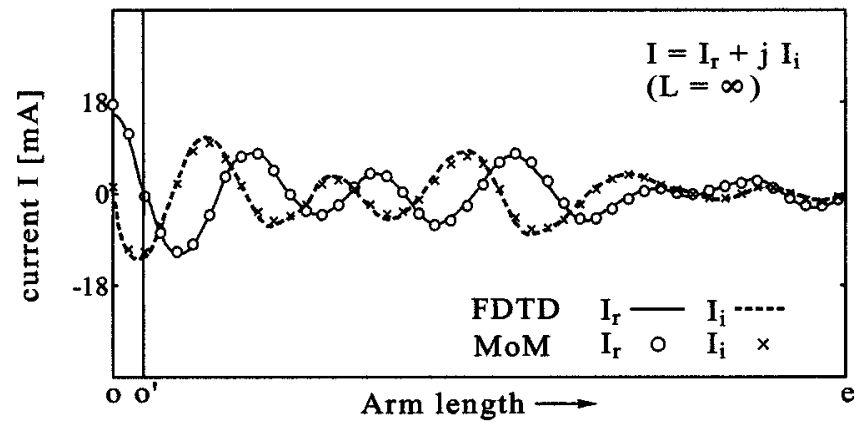

Fig. 3. Current distribution $I=I_{r}+j I_{i}$ for a spiral antenna with a $C_{2,3}$ configuration. The dielectric substrate and ground plane are of infinite extent: $L=\infty$. Analysis is performed at a test frequency of $f_{0}=3.35 \mathrm{GHz}$.

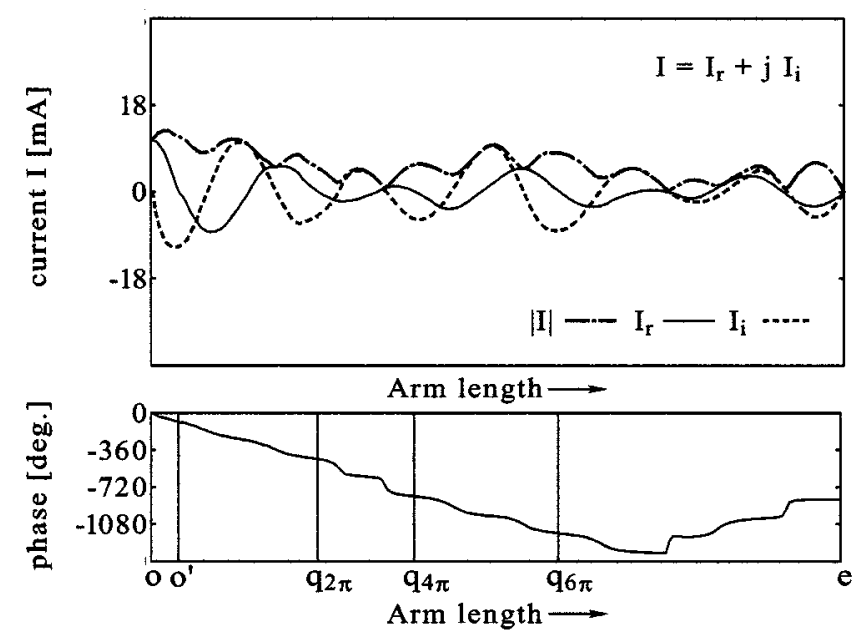

Fig. 4. Current distribution for a spiral antenna with a $C_{2,3}$ configuration. The dielectric substrate and ground plane have $L \approx 0.570 \lambda_{0}$. Analysis is performed at a test frequency of $f_{0}=3.35 \mathrm{GHz}$.

As in a round spiral antenna in free space [9], the total radiation field is characterized by the radiation fields from active regions [1]. The total radiation field of the present spiral with the $C_{2,3}$ configuration is given as the sum of the radiation field $E_{\text {first }}$ from the first active region (of an approximately $1 \lambda_{g}$ square loop on the spiral plane [1]) and the radiation field $E_{\text {second }}$ from the second active region (of an approximately $2 \lambda_{g}$ square loop on the spiral plane). The radiation fields observed at two symmetrical points with respect to the $z$-axis are nearly in-phase for $E_{\text {first }}$ and antiphase for $E_{\text {second }}$. Making use of this phase relationship between $E_{\text {first }}$ and $E_{\text {second }}$, a tilted radiation beam can be formed.

The tilted radiation beam is shown in Fig. 5(a). The radiation beam is in the $z_{1}$ direction with $\theta=34^{\circ}\left(\equiv \theta_{\max }\right)$ and $\phi=355^{\circ}\left(\equiv \phi_{\max }\right)$. Note that the $x_{1}$-axis is defined by $\phi_{\max }$, as shown in the inset of Fig. 5(d). The half-power beam width (HPBW) is approximately $82^{\circ}$ in the $x_{1}-z$ plane. The gain in the $z_{1}$ direction is $7.1 \mathrm{~dB}$ with an axial ratio of approximately $2 \mathrm{~dB}$.

Fig. 5(b) shows the radiation beam cut in the $y_{1}-z_{1}$ plane. The $y_{1}-z_{1}$ plane is perpendicular to the $x_{1}-z_{1}$ plane. The radiation pattern at $\theta=\theta_{\max }$ as a function of $\phi$ is also shown in Fig. 5(c). These patterns reveal that a right-hand circularly polarized wave is radiated around the $z_{1}$-axis. 


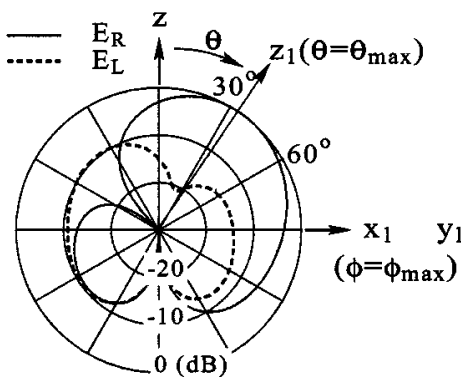

(a)

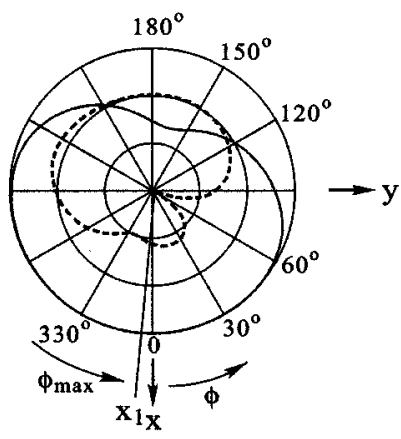

(a)

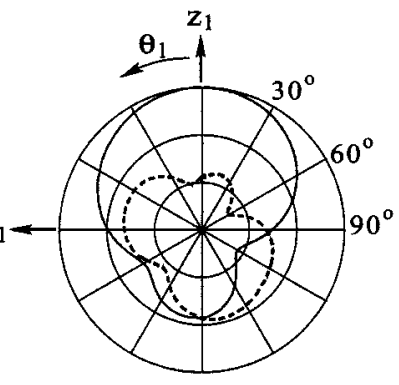

(b)

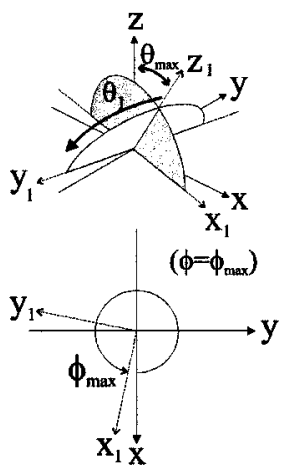

(b)
Fig. 5. Radiation patterns for a spiral antenna with a $C_{2,3}$ configuration. The dielectric substrate and ground plane have $L \approx 0.570 \lambda_{0}$. (a) $x_{1}-z$ plane, (b) $y_{1}-z_{1}$ plane, (c) $\phi$-variation at $\theta=\theta_{\max } \equiv 34^{\circ}$, and (d) definitions of $x_{1}, y_{1}$, and $z_{1}$ axes. Analysis is performed at a test frequency of $f_{0}=3.35 \mathrm{GHz}$.

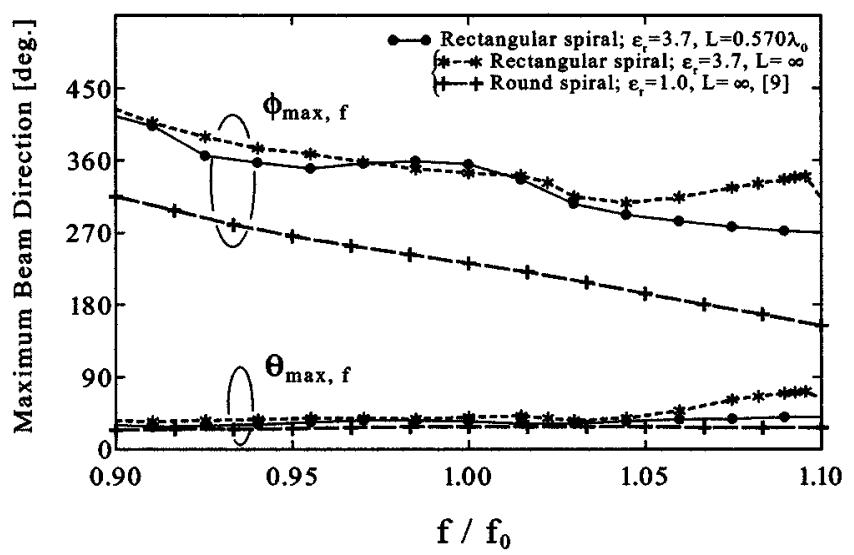

Fig. 6. Frequency response of the maximum beam direction $\left(\theta_{\max , f}, \phi_{\max , f}\right)$. The dielectric substrate and ground plane have $L \approx 0.570 \lambda_{0}$.

Further calculations are performed to reveal the frequency responses for the axial ratio and gain in the $z_{1}$ direction $\left(\theta_{\max }=\right.$ $\left.34^{\circ}, \phi_{\max }=355^{\circ}\right)$. The bandwidth for a $3-\mathrm{dB}$ axial ratio criterion is evaluated to be $6.5 \%$. This bandwidth is narrower than that of a round spiral antenna in free space [9]. The reason is that the substrate used for the rectangular spiral under consideration has a higher dielectric constant of $\varepsilon_{r}=3.7$, compared with the air dielectric substrate used for the round spiral [9]. (In general, as the dielectric constant increases, the axial ratio bandwidth of circular polarization becomes narrower [20].)

Over the above-mentioned $6.5 \%$ axial ratio bandwidth, the gain $G_{R}$ variation is small $(1.1 \mathrm{~dB})$ with a maximum gain of

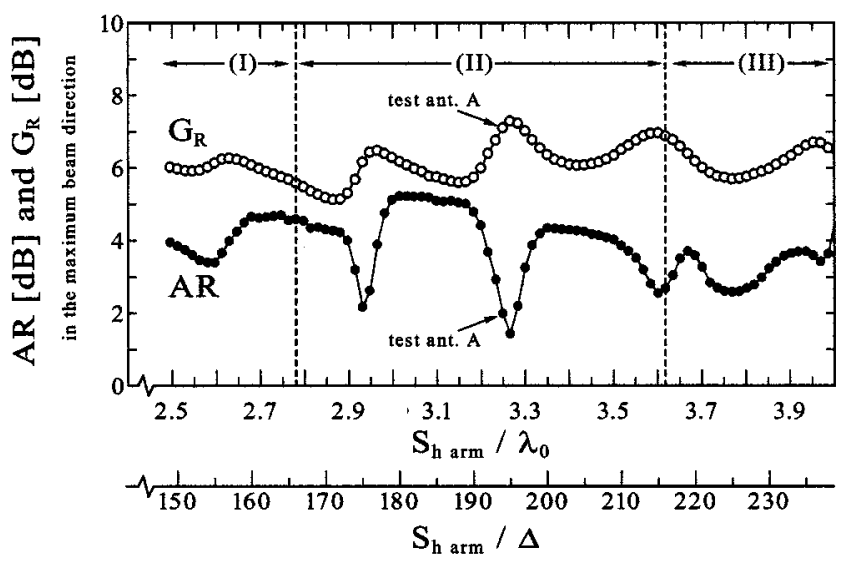

Fig. 7. AR and gain $G_{R}$ in the maximum beam direction as a function of the horizontal arm length $S_{h}$ arm. The dielectric substrate and ground plane have $L \approx 0.570 \lambda_{0}$. Analysis is performed at a test frequency of $f_{0}=3.35 \mathrm{GHz}$.

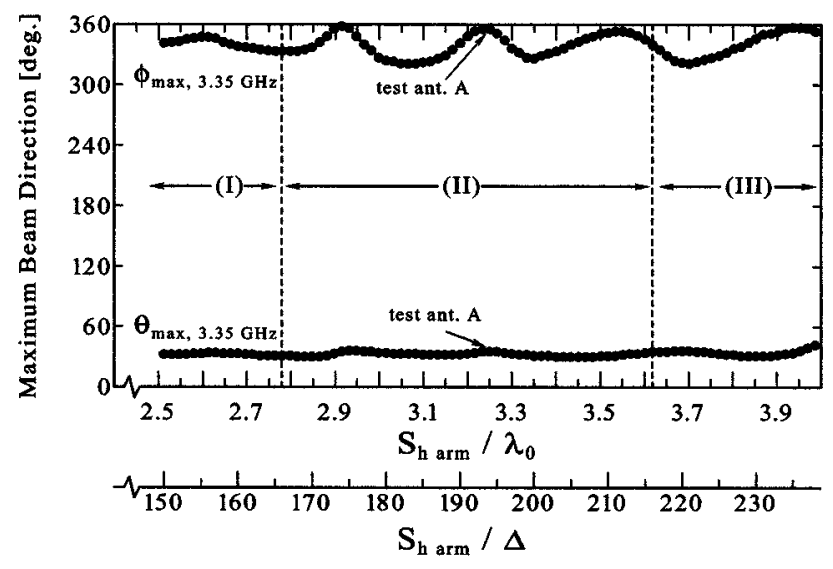

Fig. 8. Maximum beam direction $\left(\theta_{\max }, 3.35 \mathrm{GHz}, \phi_{\max }, 3.35 \mathrm{GHz}\right)$ as a function of $S_{h \mathrm{arm}}$. The dielectric substrate and ground plane have $L \approx 0.570 \lambda_{0}$. Analysis is performed at a test frequency of $f_{0}=3.35 \mathrm{GHz}$.

$G_{R} \approx 7.2 \mathrm{~dB}$. This maximum gain is lower than that for the round spiral antenna with a ground plane of infinite extent [9]. The lower gain is attributed to the finite substrate and ground plane, which allow the rectangular spiral to radiate in the negative $z$-region, forming a wider radiation beam than that of the round spiral, as shown in Fig. 5.

The VSWR (relative to the resistive component ( 92.5 $\Omega$ ) of the input impedance observed at a test frequency of $f_{0}=3.35 \mathrm{GHz}$ ) is less than two over the above-mentioned $6.5 \%$ axial ratio bandwidth, as desired.

The black dotted line in Fig. 6 shows the frequency response of the beam direction, specified by angles $\theta=\theta_{\max , f}$ and $\phi=\phi_{\max , f}$. It is found that angle $\phi_{\max , f}$ changes as the frequency varies, while angle $\theta_{\max , f}$ remains relatively constant. This behavior is also found in the case where the ground plane of finite extent is replaced with a ground plane of infinite extent $(L=\infty)$, as shown by the * line. In addition, similar beam behavior (relatively constant $\theta_{\max , f}$ and changing $\phi_{\max , f}$ as a function of frequency) is revealed for the round spiral antenna [9], as shown by the + line in Fig. 6.

The above-mentioned discussions are for a spiral antenna with a $C_{2,3}$ configuration having a horizontal arm length of $S_{h \text { arm }} \approx 3.25 \lambda_{0}$ [from point $o^{\prime}$ to point $e$ in Fig. 1(b)] and 
an outermost peripheral length of $C \approx 2.47 \lambda_{g \text {, rough. }}$. For convenience, this antenna is designated as test antenna A. In addition to the radiation characteristics of test antenna $\mathrm{A}$, those of spiral antennas with $C_{2,3}$ configurations having different horizontal arm lengths $S_{h}$ arm are briefly discussed in the following paragraph.

Fig. 7 shows the axial ratio and gain as a function of $S_{h}$ arm at $f_{0}=3.35 \mathrm{GHz}$. The arm length is expressed in terms of both the free-space wavelength $\lambda_{0}$ and the grid cell size $\Delta(=1.5 \mathrm{~mm})$. The horizontal arm length varies more than one wavelength in terms of the free-space wavelength, corresponding to more than two wavelengths in terms of the roughly estimated guided wavelength: $2.5 \lambda_{0} \leq S_{h \text { arm }} \leq 4 \lambda_{0}$ and $3.83 \lambda_{g \text {, rough }} \leq S_{h \text { arm }} \leq$ $6.13 \lambda_{g}$, rough. Three regions I, II, and III in Fig. 7 have different outermost peripheral lengths: $C=4 M a_{0} \approx 2.16 \lambda_{g}$, rough in region I $(M=7), C \approx 2.47 \lambda_{g}$, rough in region II $(M=8)$, to which test antenna A belongs, and $C \approx 2.77 \lambda_{g}$, rough in region III $(M=9)$. It is found that the minimum axial ratio and the highest gain are obtained when the horizontal arm length $S_{h}$ arm is very close to that of test antenna A. Note that the axial ratio and gain in Fig. 7 are evaluated in the maximum beam direction at $3.35 \mathrm{GHz}$, which is shown in Fig. 8. The effects of the horizontal arm length $S_{h}$ arm on the beam direction is small: $\theta_{\max , 3.35 \mathrm{GHz}}=33^{\circ} \pm 3^{\circ}$ and $\phi_{\max }, 3.35 \mathrm{GHz}=340^{\circ} \pm 18^{\circ}$.

\section{B. Spiral With a $C_{1,2}$ Configuration (Axial-Beam Spiral)}

The number of the arm filaments is further reduced to realize a $C_{1,2}$ configuration. $M=4\left(M a_{0}=12 \Delta\right)$ and $S=3$ $\left(S a_{0}=9 \Delta\right)$ are assigned to the filament parameters. The peripheral length is now $C=4 M a_{0} \approx 0.804 \lambda_{0} \approx 1.23 \lambda_{g}$, rough. The side length of the dielectric substrate is chosen to be $L=$ $22 \Delta=33 \mathrm{~mm} \approx 0.369 \lambda_{0}$, which results in a more compact structure than the previous antenna with the $C_{2,3}$ configuration. The other configuration parameters have been defined in Section II.

Fig. 9 shows the current distribution. As in the tilted beam spiral described in the previous section, a travelling-wave current flows along the spiral arm. The phase change from point o' to point $q_{2 \pi}$ is $2 \pi \mathrm{rad}$. This change defines the guided wavelength $\lambda_{g}$ as $62.3 \mathrm{~mm}$, which leads to $\lambda_{0} / \lambda_{g} \approx 89.6 \mathrm{~mm} / 62.3 \mathrm{~mm} \approx 1.44$. The peripheral length, therefore, is $C=4 M a_{0} \approx 0.804 \lambda_{0} \approx 1.16 \lambda_{g}$ and satisfies the condition for the $C_{1,2}$ configuration. Note that the ratio $\lambda_{g} / \lambda_{g}$, rough is 1.07 and hence the $\lambda_{g}$, rough again gives good prediction of the guided wavelength $\lambda_{g}$, as in the tilted beam spiral.

The spiral with the $C_{1,2}$ configuration has a first active region, i.e., the approximately $1 \lambda_{g}$ square loop region on the spiral plane [1]. Hence it is expected that radiation will occur around the $z$-axis. Fig. 10 shows the radiation patterns in the $\phi=0^{\circ}$, $45^{\circ}, 90^{\circ}$, and $135^{\circ}$ planes. Experimental results are also presented, showing good agreement with theoretical radiation patterns. As expected, the spiral radiates around the $z$-axis. This antenna, therefore, is called an "axial-beam spiral" in contrast to the "tilted-beam spiral" in the previous section.

The radiation pattern for the axial-beam spiral is not perfectly symmetrical with respect to the $z$-axis due to the fact that the spiral arm on the dielectric is not symmetrical with respect to

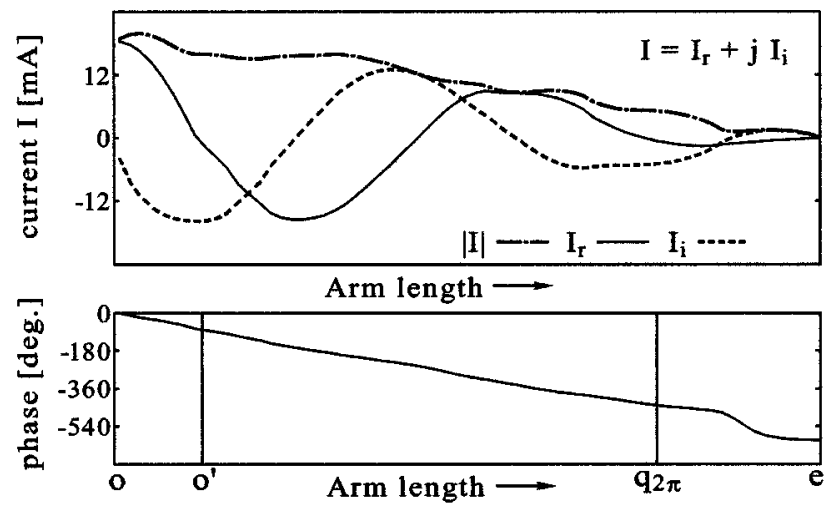

Fig. 9. Current distribution $I=I_{r}+j I_{i}$ for a spiral antenna with a $C_{1,2}$ configuration. The dielectric substrate and ground plane have $L \approx 0.369 \lambda_{0}$. Analysis is performed at a test frequency of $f_{0}=3.35 \mathrm{GHz}$.

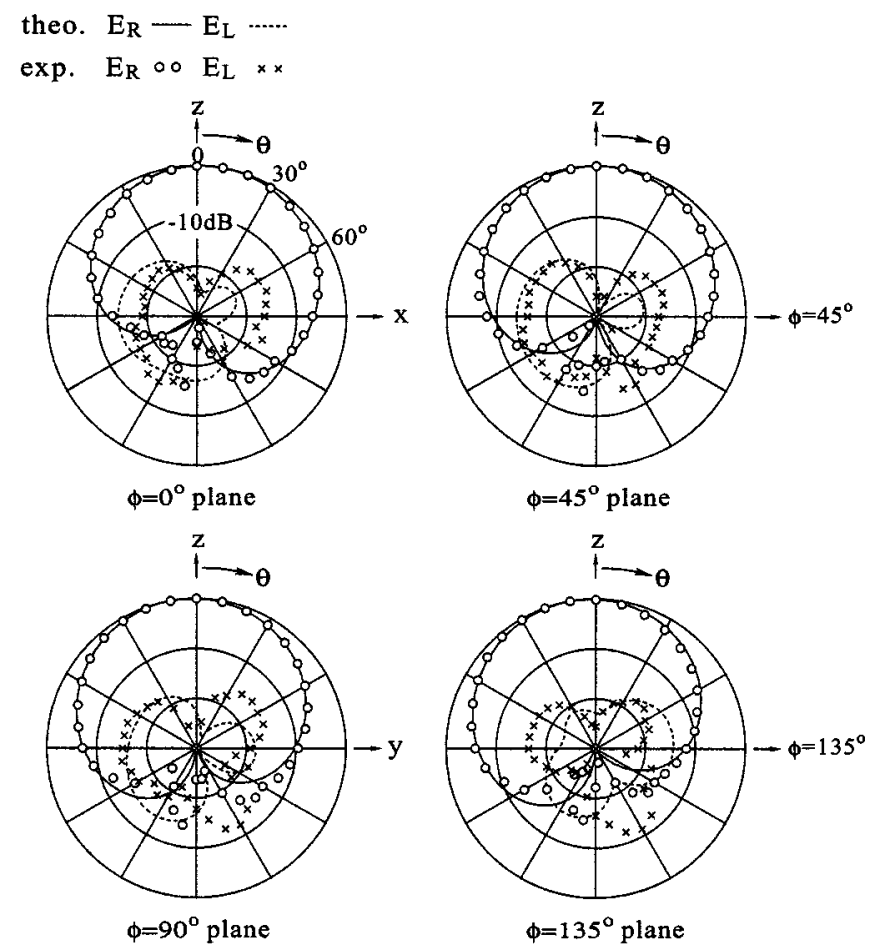

Fig. 10. Radiation patterns for a spiral antenna with a $C_{1,2}$ configuration. The dielectric substrate and ground plane have $L \approx 0.369 \lambda_{0}$. Analysis is performed at a test frequency of $f_{0}=3.35 \mathrm{GHz}$.

the $z$-axis. The average value of the HPBW in the four planes in Fig. 10 is approximately $102^{\circ}$. The radiation on the $z$-axis is circularly polarized with an axial ratio of $1.3 \mathrm{~dB}$, and the gain on the $z$-axis is approximately $6.7 \mathrm{~dB}$ (slightly lower than the gain for the tilted-beam spiral).

Fig. 11 shows the maximum beam direction $\left(\theta_{\max , f}, \phi_{\max , f}\right)$ as a function of frequency. As seen from $\theta_{\max , f}$, the axial beam radiation is maintained. Fig. 12 shows the frequency responses of the $\mathrm{AR}$ and gain $G_{R}$ in the $z$ direction. Experimental results are also presented with black and white dots. The theoretical data agree well with the experimental results. The bandwidth for a $3-\mathrm{dB}$ axial ratio criterion is evaluated to be approximately $15 \%$, which is more than two times wider than the bandwidth for the tilted-beam 


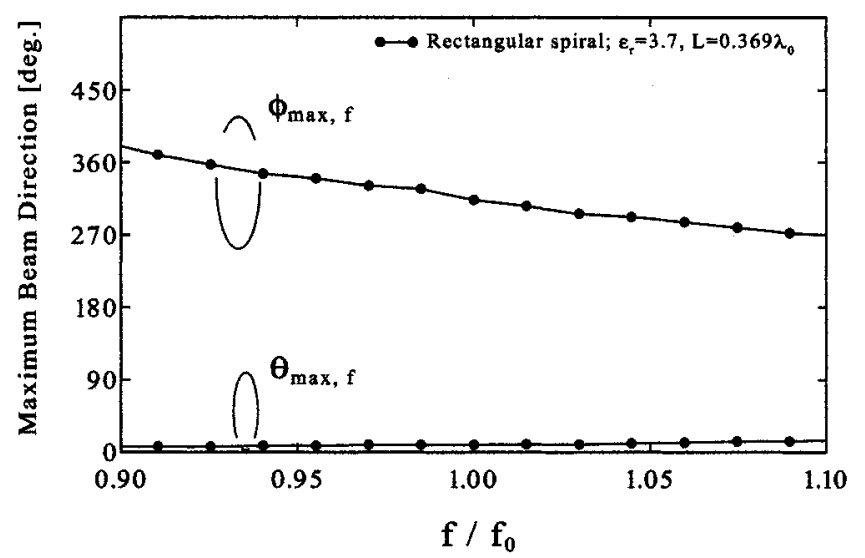

Fig. 11. Frequency response of the maximum beam direction $\left(\theta_{\max , f}, \phi_{\max , f}\right)$. The dielectric substrate and ground plane have $L \approx 0.369 \lambda_{0}$

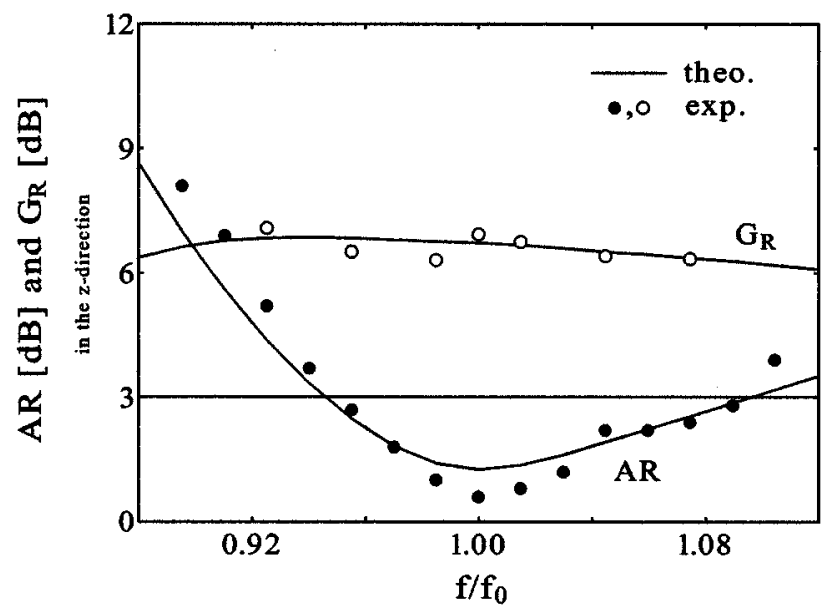

Fig. 12. Frequency responses of $\mathrm{AR}$ and $G_{R}$ in the $z$ direction. The spiral antenna has a $C_{1,2}$ configuration. The dielectric substrate and ground plane have $L \approx 0.369 \lambda_{0}$.

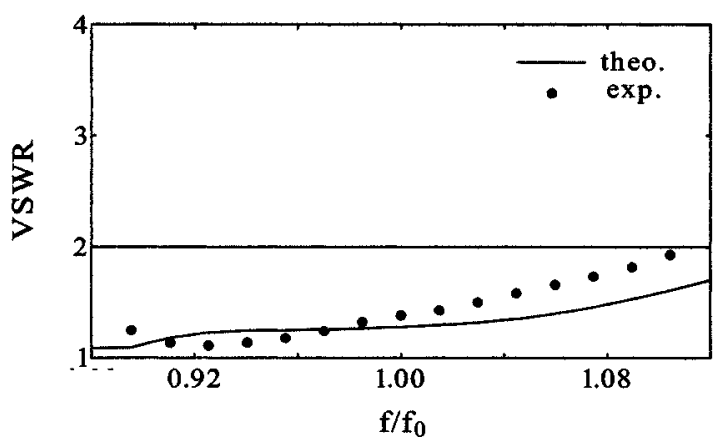

Fig. 13. Frequency response of the VSWR for a spiral antenna with a $C_{1,2}$ configuration. The dielectric substrate and ground plane have $L \approx 0.369 \lambda_{0}$.

spiral (and for the curl antenna [21], which also radiates an axial beam of circular polarization). The variation in $G_{R}$ over this bandwidth is very small $(0.6 \mathrm{~dB})$.

Fig. 13 presents the VSWR relative to the resistive component $\left(51.6 \Omega\right.$ ) of the input impedance observed at $f_{0}=3.35 \mathrm{GHz}$. Desirable VSWRs of less than two are obtained in this analysis

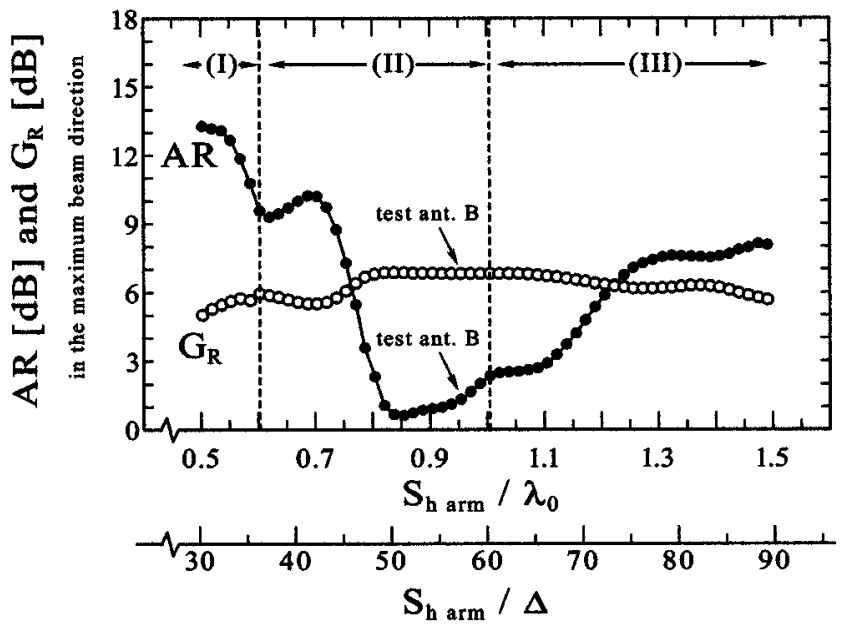

Fig. 14. AR and $G_{R}$ in the maximum beam direction as a function of the horizontal arm length $S_{h}$ arm. The dielectric substrate and ground plane have $L \approx 0.369 \lambda_{0}$. Analysis is performed at a test frequency of $f_{0}=3.35 \mathrm{GHz}$.

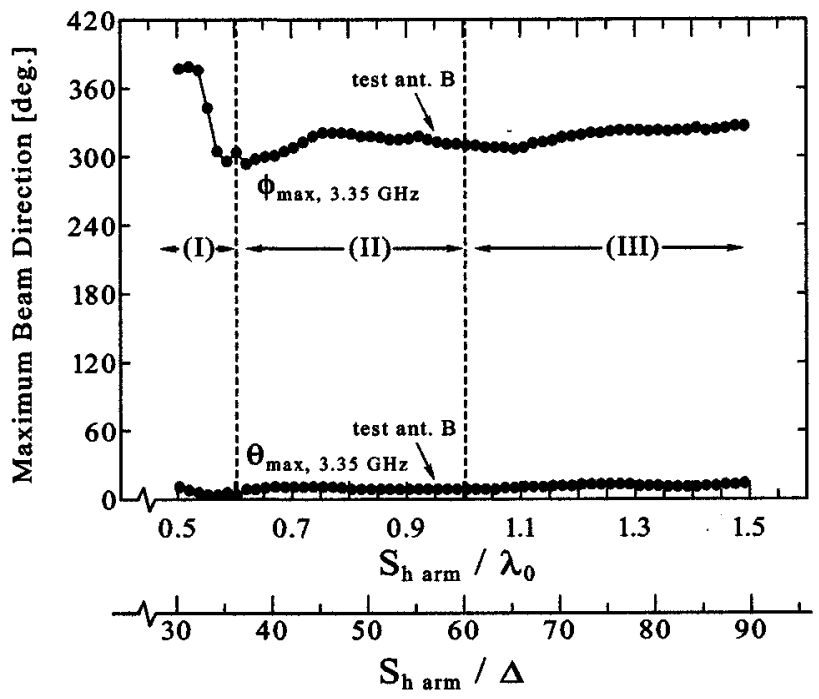

Fig. 15. Maximum beam direction $\left(\theta_{\max }, 3.35 \mathrm{GHz}, \phi_{\max }, 3.35 \mathrm{GHz}\right)$ as a function of $S_{h \mathrm{arm}}$. The dielectric substrate and ground plane have $L \approx 0.369 \lambda_{0}$. Analysis is performed at a test frequency of $f_{0}=3.35 \mathrm{GHz}$.

range. Note that experimental and theoretical results are in good agreement.

So far, a spiral antenna having a peripheral length of $C \approx$ $1.16 \lambda_{g}$, which leads to an example of the $C_{1,2}$ configuration, has been analyzed. For convenience, this antenna is designated as test antenna B. Fig. 14 shows a range of axial ratio and gain values, including those of test antenna $\mathrm{B}$, at $f_{0}=3.35 \mathrm{GHz}$. The peripheral length of the spiral in these figures is less than two wavelengths in terms of the roughly estimated guided wavelength: $C=4 M a_{0} \approx 0.924 \lambda_{g}$, rough in region I $(M=3)$, $C \approx 1.23 \lambda_{g, \text { rough }}$ in region II $(M=4)$, and $C \approx 1.54 \lambda_{g, \text { rough }}$ in region III $(M=5)$. Note that the axial ratio and gain in Fig. 14 are evaluated in the maximum beam direction at 3.35 $\mathrm{GHz}$, which is shown in Fig. 15. It is noted that:

1) the horizontal arm length chosen for test antenna B realizes a good axial ratio value for a circularly polarized wave radiator; 
2) the radiation forms an axial beam with a slight deviation from the $z$-axis $\left(\theta_{\max }, 3.35 \mathrm{GHz}\right.$ deviates approximately $9^{\circ}$, due to the asymmetrical antenna configuration with respect to the $z$-axis);

3 ) the gain is relatively constant in the vicinity of the gain for test antenna B.

\section{CONCLUSION}

The characteristics of a rectangular spiral antenna printed on a dielectric substrate backed by a conducting plane have been considered under the conditions that the substrate and conducting plane are of finite size and are square with side length $L$. The FDTD method is used for analysis. The FDTD analysis result (the current distribution for a special case in which the conducting plane and the dielectric substrate are of infinite extent, i.e., $L=\infty$ ) has been validated by good agreement with the MoM analysis result. Based on this validation, investigation into the $C_{1,2}$ and $C_{2,3}$ configurations has been performed using an $L$ of less than 0.6 wavelengths to make the configuration compact.

The spiral with the $C_{2,3}$ configuration, in which the outermost peripheral length $C$ is within $2 \lambda_{g}<C<3 \lambda_{g}$, radiates a tilted beam of circular polarization. A tilt angle of approximately $\theta_{\max }=34^{\circ}$ is obtained with a half-power beam width of $82^{\circ}$. The frequency bandwidth for a $3-\mathrm{dB}$ axial ratio criterion (in the direction of $\theta_{\max }=34^{\circ}$ and $\phi_{\max }=355^{\circ}$ ) is evaluated to be $6.5 \%$. Over this bandwidth, the gain variation from a maximum value of $G_{R} \approx 7.2 \mathrm{~dB}$ is small $(1.1 \mathrm{~dB})$ and the VSWR is less than two, as desired.

The spiral with the $C_{1,2}$ configuration, in which the outermost peripheral length $C$ is within $\lambda_{g}<C<2 \lambda_{g}$, shows a decaying current distribution along the spiral arm, which contributes to forming an axial beam of circular polarization. The analysis has revealed that the axial beam has a wide half-power beam width of approximately $102^{\circ}$ with a gain of approximately $6.7 \mathrm{~dB}$ (on the $z$-axis) at a test frequency of $f_{0}=3.35 \mathrm{GHz}$. It has also been found that the frequency bandwidth for a $3-\mathrm{dB}$ axial ratio criterion (on the $z$-axis) is approximately $15 \%$, over which desirable VSWRs of less than two are obtained. The FDTD analysis results for the radiation pattern, gain, axial ratio, and VSWR are in good agreement with experimental results.

\section{ACKNOWLEDGMENT}

The authors would like to thank V. Shkawrytko for his assistance in the preparation of this manuscript.

\section{REFERENCES}

[1] J. A. Kaiser, "The Archimedean two-wire spiral antenna," IRE Trans. Antennas Propagat., vol. AP-8, pp. 312-323, May 1960.

[2] H. Nakano, K. Nogami, S. Arai, H. Mimaki, and J. Yamauchi, "A spiral antenna backed by a conducting plane reflector," IEEE Trans. Antennas Propagat., vol. AP-34, pp. 791-796, June 1986.

[3] H. Nakano, S. R. Kerner, and N. G. Alexopoulos, "The moment method solution for printed wire antennas of arbitrary configuration," IEEE Trans. Antennas Propagat., vol. 36, pp. 1667-1674, Dec. 1988.

[4] J. J. H. Wang and V. K. Tripp, "Design of multioctave spiral-mode microstrip antennas," IEEE Trans. Antennas Propagat., vol. 39, pp. 332-335, Mar. 1991.

[5] L. Shafai, "Design of multi-arm multi-mode spiral antennas for directional beams using equivalent array concept," Electromagnetics, vol. 14, no. 3-4, pp. 285-304, July-Dec. 1994.

[6] P. E. Mayes, "Planar and other wide-angle logarithmic spirals over ground," Electromagnetics, vol. 14, no. 3-4, pp. 329-362, July-Dec. 1994.

[7] N. J. Champagne II, J. T. Williams, and D. R. Wilton, "Analysis of resistively loaded, printed spiral antennas," Electromagnetics, vol. 14, no. 3-4, pp. 363-395, July-Dec. 1994.

[8] U. R. Kraft, "Polarization properties of small printed spiral antennas with four resistively loaded arms," Inst. Elect. Eng. Proc. Microwave Antennas Propag., vol. 144, no. 2, pp. 131-135, Apr. 1997.

[9] H. Nakano, Y. Shinma, and J. Yamauchi, "A monofilar spiral antenna and its array above a ground plane-Formation of a circularly polarized tilted fan beam," IEEE Trans. Antennas Propagat., vol. 45, pp. 1506-1511, Oct. 1997.

[10] J. Chen, A. Z. Elshebeni, C. E. Smith, and Y. Rahamat-Samii, "FDTD analysis of printed square spiral antennas for wireless communications," in IEEE Antennas Propagation Int. Symp. Dig., vol. 3, Montreal, PQ, July 1997 , pp. 1550-1553.

[11] T. Ozdemir, M. W. Nurnberger, and J. Volakis, "A thin cavity-backed Archimedean slot spiral for 800-3000 MHz band coverage," in IEEE Antennas Propagation Int. Symp. Dig., vol. 4, Atlanta, June 1998, pp. 2336-2339.

[12] E. Yamashita, Analysis Methods for Electromagnetic Wave Problems, E. Yamashita, Ed. Boston, MA: Artech House, 1996, vol. 2.

[13] R. F. Harrington, Field Computation by Moment Methods. New York: Macmillan, 1968.

[14] S. K. Khamas, G. G. Cook, R. J. Waldron, and R. M. Edwards, "Moment method analysis of printed single-arm wire spiral antennas using curved segments," Inst. Elect. Eng. Proc. Microwave Antennas Propagation, vol. 144, no. 4, pp. 261-265, August 1997.

[15] R. L. Lin and H. Nakano, "Numerical analysis of arbitrarily shaped probe-excited single-arm printed wire antennas," IEEE Trans. Antennas Propagat., vol. 46, pp. 1307-1317, Sept. 1998.

[16] K. S. Yee, "Numerical solution of initial boundary value problems involving Maxwell's equations in isotropic media," IEEE Trans. Antennas Propagat., vol. AP-14, pp. 302-307, May 1966.

[17] A. Taflove, Computational Electrodynamics. Norwood, MA: Artech House, 1995.

[18] Z. P. Liao, H. L. Wong, B. P. Yang, and Y. F. Yuan, "A transmitting boundary for transient wave analysis," Sci. Sinica A, vol. 27, no. 10, pp. 1063-1076, 1984.

[19] T. Uno, "Field and antenna analysis by FDTD method," Tutorial Text, Institute of Electronics, Information and Communications, Japan, 1996.

[20] Y. Nakamura, J. Yamauchi, and H. Nakano, "Strip loop antenna with an electromagnetically coupled feed system," in Proc. 2001 IEICE General Conf., Shiga, Japan, Mar. 2001, pp. B1-149.

[21] H. Nakano, S. Okuzawa, K. Ohishi, H. Mimaki, and J. Yamauchi, "A curl antenna," IEEE Trans. Antennas Propagat., vol. 41, pp. 1570-1575, Nov. 1993. 